\title{
DESIGN CONSIDERATIONS FOR SYMMETRICALLY LAMINATED PLATES WITH CUTOUTS
}

\author{
E. Senocak* and A. M. Waas ${ }^{\dagger}$ \\ Dept. of Aerospace Engineering, Univ. of Michigan, Ann Arbor, MI 48109-2140
}

February 1, 1992

\begin{abstract}
Earlier [1], it was shown that certain reinforced holes may be designed for elastic, isotropic plane sheets that do not alter the stress state in the cut sheet, for particular types of planar loading. In this work, this methodology is extended to symmetrically laminated composite plates which are under planar loading, and additionally pure bending moments. Since these two types of loading lead to uncoupled governing equations, they are treated separatly in two parts. The shape of the cutout and the cross-sectional area of the reinforcement are determined in closed form. The reinforcement is modeled as a one-dimensional rod/beam type structural element.
\end{abstract}

\section{Introduction}

Since laminated composite plates with openings are widely used structural elements in many engineering applications, the analysis of such structures has been done by numerous researchers. The stress and strain state around the openings have been presented for a variety of problems. A large number of problem solutions are presented in the monographs by Lekhnitskii [2],[3] and Savin [4]. In the formulation of these problems, the material constitutive law, the geometry, loading and boundary conditions are assumed given and the stress and strain state are computed.

In a particular class of structural optimization, the geometry of the body is obtained as a solution to a particular problem, for known states of stress, strain, boundary condition and appropriate constraints (sometimes called inverse problems for some specific cases).

\footnotetext{
* Graduate Research Assistant, Student Member AIAA

tAsst. Professor of Aerospace Engineering, Member AIAA. Copyright c 1992 by Anthony M. Waas. Published by the American Institute of Aeronautics and Astronautics, Inc. with permission.
}

In the latter category, either stress concentrations are reduced (optimized) by determining the shape of the openings (harmonic holes), or stress states in the cut structures with reinforcement are maintained unchanged to that of the uncut structures (neutral holes).

All previous solutions $([1],[5])$, for neutral holes have been obtained for elastic, isotropic sheets under planar states of stress. In [5], the reinforcing member is assumed to be additionally under a planar moment also, but the solution is still sought as a plane problem. In the present research, the methodology outlined in [1], is extended to symmetrically laminated composite plates which are under planar loading. In addition, the case of a symmetrically laminated plate subjected to pure bending moments is also considered. Since these two types of loadings lead to uncoupled governing equations, they are treated separatly in two parts.

In the first part, laminated plates are assumed subjected to loading that induce planar states of stress for a given symmetric lay up and loading. The shape and cross-sectional area of the reinforcement are determined in closed form. The reinforcement is assumed to be made of the ply material that is used to construct the laminate, although this assumption is not necessary. It is used here to generate example results, to be presented later. In addition, the fiber direction in the reinforcement is assumed to be tangential to the edge of the cutout. Since the sectional areas of the reinforcing member are small compared to the other dimensions of the plate, they are treated within the framework of one dimensional technical rod/beam theories.

A number of examples are solved for different fiber angles and loading conditions. Solutions in which the circumferential strain around the hole changes sign are impractical for some lay ups and fiber angles. This situation is treated by changing the fiber angle of the plates or just the outer layers. Under some types of loading, closed holes are found not to be possible and this is acknowledged here. 
In the second part, classical plate theory assumptions are employed to the pure bending problem of laminated plates. In tins case, the reinforcement is assumed as a beam element which is under torsional and bending moments. This is a new treatment of neutral hole problems in which flexural plate response is taken into account. The shape and cross-sectional area of the reinforcement are presented for two separate cases. In the first, a restriction is placed, forcing a relation between the torsional and bending moments of the reinforcing member. This is done so that the shape of the hole could be determined by considering the equations of statics alone for a small element adjacent to the cutout and containing a part of the reinforcement. Extreme cases such as a reinforcement infinitly rigid against rotation, or infinitly rigid against bending, are examined. In the second case, a solution is sought which combines the equations of equilibrium of the small element adjacent to the cutout and containg a part of the reinforcement with the kinematics of the reinforcement. This solution does not employ any simplifying assumptions and is obtained in closed form. The governing equation for this case is solved numerically, and results for the shape of the hole and reinforcement distribution are computed for example cases. Notice that the notion of a neutral hole in the context of plate flexure assumes a new meaning. Here, the purpose is to introduce a cutout into a plate that will maintain the same moment and curvature distributions as of the uncut plate throughout.

The formulation for the laminated plate presented in the first part is shown to reduce to that of the solution for the isotropic case [1], by making appropriate substitution for the elastic constants. The solutions for the second part are novel and similar issues have not been dealth with before.

\section{Formulation}

\subsection{Laminated plate under planar loading}

\subsubsection{The shape of a neutral hole}

The derivation given here follows along the lines of presentation in [1], and is given here for completness. Consider the uncut, uniformly thick, laminated plate in a planar state of stress. Let a set of cartesian coordinates be situated in the mid plane of the laminate (symmetrically laminated about the mid plane) such that the $x y$ plane coincides with the lamination plane. For a two dimensional planar state of stress, the equilibrium equations, in terms of plate resultants are

$$
\begin{aligned}
& \frac{\partial N_{x}}{\partial x}+\frac{\partial N_{x y}}{\partial y}=0 \\
& \frac{\partial N_{x y}}{\partial x}+\frac{\partial N_{y}}{\partial y}=0 .
\end{aligned}
$$

These equations are satisfied by the introduction of a stress function defined as below.

$$
\begin{aligned}
N_{x} & =\frac{\partial^{2} \phi}{\partial y^{2}} \\
N_{y} & =\frac{\partial^{2} \phi}{\partial x^{2}} \\
N_{x y} & =-\frac{\partial^{2} \phi}{\partial x \partial y}
\end{aligned}
$$

Thus, a knowledge of $\phi(x, y)$ completely specifies the planar state of stress in the plate.

With reference to Fig.1 and Fig.2, which show the cut sheet and the reinforcement, consider the equilibrium of an element adjacent to the cutout and including a part of the reinforcement. Assuming that the reinforcing member can be treated as a one dimensional rod element, the equilibrium equations are as follows.

$$
\begin{aligned}
d(P \sin \alpha) & =N_{y} d x-N_{x y} d y \\
d(P \cos \alpha) & =N_{x y} d x-N_{x} d y
\end{aligned}
$$

where $P$ is positive in tension. Upon substituting (2) into (3) the following is derived.

$$
\begin{aligned}
d(P \sin \alpha) & =\frac{\partial^{2} \phi}{\partial x^{2}} d x+\frac{\partial^{2} \phi}{\partial x \partial y} d y \\
d(P \cos \alpha) & =-\frac{\partial^{2} \phi}{\partial x \partial y} d x-\frac{\partial^{2} \phi}{\partial y^{2}} d y
\end{aligned}
$$

Integration of (4) leads to;

$$
\begin{aligned}
& P \sin \alpha=\frac{\partial \phi}{\partial x}+a \\
& P \cos \alpha=-\frac{\partial \phi}{\partial y}-b
\end{aligned}
$$

where $a$ and $b$ are integral constants. From (5) $P$ may be eliminated, by setting $\frac{d y}{d x}$ for $\tan \alpha$ (see Fig.2 where $\alpha$ is marked) and integrating to obtain,

$$
\phi+a x+b y+c=0
$$

as the equation to determine the shape of the hole.

Terms of the type $(a x+b y+c)$ do not alter the stresses, so without loss of generality (6) can be written as

$$
\phi=0 .
$$


Equation (7) is obtained purely from a consideration of static equilibrium of the small element at the hole.

\subsubsection{Section area of the reinforcement}

The total force in the reinforcing member is determined from equation (5), by eliminating $\alpha$.

$$
P=\left[\left(\frac{\partial \phi}{\partial x}\right)^{2}+\left(\frac{\partial \phi}{\partial y}\right)^{2}\right]^{\frac{1}{2}}
$$

The well known constitutive relation, derived on the basis of classical lamination plate theory (CLT) is [6],

$$
N=A \epsilon+B \kappa \text {. }
$$

For a symmetric layup $[B]=[0]$, and solving for $\epsilon$ from (9) yields

$$
\epsilon=A^{-1} N=A^{*} N \text {. }
$$

Equation (10) in expanded form is given as,

$$
\left[\begin{array}{c}
\epsilon_{x} \\
\epsilon_{y} \\
\epsilon_{x y}
\end{array}\right]=\left[\begin{array}{lll}
A_{11}{ }^{*} & A_{12}{ }^{*} & A_{16}{ }^{*} \\
A_{12}{ }^{*} & A_{22}{ }^{*} & A_{26}{ }^{*} \\
A_{16}{ }^{*} & A_{26}{ }^{*} & A_{66}{ }^{*}
\end{array}\right]\left[\begin{array}{c}
N_{x} \\
N_{y} \\
N_{x y}
\end{array}\right] .
$$

Thus, equation (11) describes the strain state in the plate in terms of plate stress resultants. By making use of equation (2), the relation between the stress function $\phi(x, y)$ and the strain state is obtained via (10). The strains in the plate are continuous and therefore the tangential component of strain at the hole edge (i.e.,along the reinforcement) is given by the strain transformation law

$$
\bar{\epsilon}_{s}=\epsilon_{x} \cos ^{2} \alpha+\epsilon_{y} \sin ^{2} \alpha+\epsilon_{x y} \sin \alpha \cos \alpha .
$$

Finally, the stress resultant in the reinforcing member, using a $1 \mathrm{D}$ relation, may be written as,

$$
\bar{N}=\bar{A}_{s s} \bar{\epsilon}_{s} \text {. }
$$

Here, $\bar{A}_{s s}$ is the axial stiffness of the reinforcement. But,

$$
\bar{N}=\frac{P}{d}
$$

where $d$ is the width of the reinforcement. Using (8), (13) and (14) the width distribution of the reinforcement is obtained as,

$$
d(s)=\frac{\left[\left(\frac{\partial \phi}{\partial x}\right)^{2}+\left(\frac{\partial \phi}{\partial y}\right)^{2}\right]^{\frac{1}{2}}}{\bar{A}_{s s} \bar{\epsilon}_{s}} .
$$

\subsection{Laminated plate under bending}

\subsubsection{The shape of the hole}

Let a cartesian coordinate system be located in the mid plane of the laminate, about which the plate is laminated symmetrically, such that the $x y$ plane coincides with the lamination plane and the $z$ axis is perpendicular to that plane. Under Classical Laminate Theory assumptions, the lateral force and moment equilibrium equations are the following [7],

$$
\begin{aligned}
\frac{\partial Q_{x}}{\partial x}+\frac{\partial Q_{y}}{\partial y}+q & =0 \\
\frac{\partial M_{x}}{\partial x}+\frac{\partial M_{x y}}{\partial y} & =Q_{x} \\
\frac{\partial M_{y}}{\partial y}+\frac{\partial M_{x y}}{\partial x} & =Q_{y} .
\end{aligned}
$$

Here, $M_{x}, M_{y}$ and $M_{x y}$ are the moment resultants (moment per unit length), $Q_{x}, Q_{y}$ are the shear forces per unit length, and $q$ is the lateral surface force per unit area acting on the laminate. From (16) the following can be deduced in the absence of a lateral force $q$. In this way, the equilibrium is represented with one equation.

$$
\frac{\partial^{2} M_{x}}{\partial x^{2}}+\frac{\partial^{2} M_{y}}{\partial y^{2}}+2 \frac{\partial^{2} M_{x y}}{\partial x \partial y}=0
$$

The reinforcing member is assumed as a beam element which has flexural as well as torsional rigidity. With reference to Fig. 3 and Fig. 4 the out-of-plane force and moment equlibrium equations for a small portion of the laminate adjacent to the cutout and including the reinforcement are,

$$
\begin{aligned}
d Q & =Q_{y} d x-Q_{x} d y \\
d(T \cos \alpha+M \sin \alpha) & =M_{y} d x+\left(Q-M_{x y}\right) d y(18) \\
d(T \sin \alpha-M \cos \alpha) & =M_{x} d y+\left(-M_{x y}-Q\right) d x
\end{aligned}
$$

where $M$ and $T$ are respectively the total bending and torsional moments in the reinforcement, and $Q$ is the total shearing force in the reinforcement.

To proceed with the solution of (18), first consider (17) and introduce the functions $\varphi$ and $\psi$ defined as below, which satisfy the equlibrium equation (17).

$$
\begin{aligned}
M_{y} & =\frac{\partial \varphi}{\partial x} \\
Q-M_{x y} & =\frac{\partial \varphi}{\partial y} \\
M_{x} & =\frac{\partial \psi}{\partial y}
\end{aligned}
$$




$$
-M_{x y}-Q=\frac{\partial \psi}{\partial x}
$$

From (19) and (20) $M_{x y}$ and $Q$ are to be found as;

$$
\begin{aligned}
M_{x y} & =-\frac{1}{2}\left(\frac{\partial \varphi}{\partial y}+\frac{\partial \psi}{\partial x}\right) \\
Q & =\frac{1}{2}\left(\frac{\partial \varphi}{\partial y}-\frac{\partial \psi}{\partial x}\right) .
\end{aligned}
$$

Upon substituting (19) and (20) into (18), and then integration of the last two moment equations gives the following.

$$
\begin{aligned}
& T \sin \alpha-M \cos \alpha=\psi+e \\
& T \cos \alpha+M \sin \alpha=\varphi+f
\end{aligned}
$$

where $e$ and $f$ are constants of integration.

An immediate result of equation $(23)$ is $M^{2}+T^{2}=$ $(\psi+e)^{2}+(\varphi+f)^{2}$. Thus, the Equivalent bending moment $W$ is the vectoral sum of $M$ and $T$ at each point in the reinforcement, and can be written as,

$$
W^{2}=M^{2}+T^{2} .
$$

Next, consider a cross-section normal to the axial direction of the reinforcement. Let $\theta$ be the angle between $W$ and this cross-section (see Fig.5). Then, the following can be written.

$$
\begin{aligned}
M & =W \cos \theta \\
T & =W \sin \theta
\end{aligned}
$$

where $\theta$ describes the orientation of $W$ along different axial positions of the reinforcement.

Since $\theta$ is not known, a condition for the neutral hole cannot be deduced from equilibrium equations alone, unless some additional restrictions are enforced; (such as fixing the angle $\theta$ ). Thus, to obtain an exact solution for the shape of the hole and the reinforcement distribution, kinematic relations governig the deformation of the reinforcement have to be used. However, combining infromation between the kinematic relations and the equilibrium equation leads to a highly nonlinear equation for the determination of neutral holes. The solution of this equation which leads to a closed form solution for the hole shape and the reinforcement distribution is given later in section (2.3).

First, we seek an approximate solution for the hole shape. As noted earlier, the reinforcing edge beams around cutouts, which provide a flexible support, are assumed to resist the plate deformations by virtue of their flexural and torsional stiffnesses. In practice, the torsional stiffness is usually much less than the beam's flexural stiffness. Using (25), a connection between the torsional and bending moments developed in the reinforcement can be a priori prescribed. Then, with small $\theta$ values weak torsional resistances can be recovered.

From equation (25) $\tan \theta=T / M$ is obtained. For a fixed value of $\theta$, introduce a scalar number $\lambda$ such that $\tan \theta=\lambda$ (Here, we are prescribing a relation between $T$ and $M$, thus placing a restriction on the orientation of $W$ ).

Then, from equation (23), and setting $\frac{d y}{d x}$ for $\tan \alpha$, the following is derived.

$\lambda \psi d x+\psi d y-\lambda \varphi d y+\varphi d x=\lambda f d y-\lambda e d x-e d y-f d x$

Equation (26) gives the condition of the shape of the hole in terms of the known functions $\psi$ and $\varphi$, for a known value of $\lambda$.

2.2.2. Sectional area of the reinforcing member The total bending moment in the reinforcement is determined from equation (23) by eliminating $\alpha$.

$$
M=\left[\frac{(\psi+e)^{2}+(\varphi+f)^{2}}{1+\lambda^{2}}\right]^{\frac{1}{2}}
$$

In this case, the constitutive relation $M=B \epsilon+D \kappa$ is utilised, and by setting $[B]=[0]$ for symmetric layups, $\kappa$ is solved as,

$$
\kappa=D^{-1} M=D^{*} M .
$$

In expanded form, equation (28) is the following.

$$
\left[\begin{array}{c}
\kappa_{x} \\
\kappa_{y} \\
\kappa_{x y}
\end{array}\right]=\left[\begin{array}{lll}
D_{11}{ }^{*} & D_{12}{ }^{*} & D_{16}{ }^{*} \\
D_{12}{ }^{*} & D_{22}{ }^{*} & D_{26}{ }^{*} \\
D_{16}{ }^{*} & D_{26}{ }^{*} & D_{66}{ }^{*}
\end{array}\right]\left[\begin{array}{c}
M_{x} \\
M_{y} \\
M_{x y}
\end{array}\right]
$$

Since the curvatures in the plate are also continuous, the tangential and twisting components of the curvature are given by the curvature transformation law $[8]$,

$$
\begin{aligned}
\bar{\kappa}_{s}= & \kappa_{x} \cos ^{2} \alpha+\kappa_{y} \sin ^{2} \alpha+2 \kappa_{x y} \sin \alpha \cos \alpha \\
\bar{\kappa}_{s n}= & \left(\kappa_{y}-\kappa_{x}\right) \sin \alpha \cos \alpha+ \\
& \kappa_{x y}\left(\cos ^{2} \alpha-\sin ^{2} \alpha\right)
\end{aligned}
$$

The bending moment resultant in the reinforcement may be given as,

$$
\bar{M}=\bar{D}_{s s} \bar{\kappa}_{s}
$$

where $\bar{D}_{s s}$ is the bending stiffness of the reinforcing member. But,

$$
\vec{M}=\frac{M}{\bar{d}}
$$


where $\bar{d}$ is the width of the reinforcement in this case.

Finally, the combination of (27), (31) and (32) gives the reinforcement width distribution as,

$$
\bar{d}(s)=\frac{\left[\frac{(\psi+e)^{2}+(\varphi+f)^{2}}{1+\lambda^{2}}\right]^{\frac{1}{2}}}{\bar{D}_{s s} \bar{\kappa}_{s}} .
$$

\subsection{Plate bending solution including reinforce- ment kinematics}

In this section, we present the more complicated but exact solution for the shape of the hole and reinforcement distribution incorporating kinematics of the reinforcing member. An exact solution is obtained within Classical Laminate Theory assumptions. Consider a small element of laminate including a portion of the reinforcement. Then, (see Fig. 2), as before, the equilibrium of this element reduce to (23). i.e.,

$$
\begin{aligned}
T & =\psi \sin \alpha+\varphi \cos \alpha \\
M & =\varphi \sin \alpha-\psi \cos \alpha
\end{aligned}
$$

The torsional and bending moment in the reinforcement are related to the reinforcement twist and reinforcement curvature respectively (the constitutive equations for the reinforcernent). These are,

$$
\begin{aligned}
T & =G_{n z} J \bar{\kappa}_{s n} \\
M & =E_{s} I \bar{\kappa}_{s}
\end{aligned}
$$

where $E_{s} I$ and $G_{n z} J$ are the bending and torsional rigidities of the reinforcement respectively (see Fig. 6). To proceed and demonstrate the complete solution consider a rectangular cross-section for the reinforcement. Let $h$ denote the reinforcement thickness and $d$ the width. Then, the $I$ and $J$ values are found as,

$$
\begin{aligned}
I & =\frac{d h^{3}}{12} \\
J & =\frac{0.3 h^{3} d^{3}}{h^{2}+d^{2}} .
\end{aligned}
$$

Combining equations (34),(35) and (36), the following is derived.

$$
\begin{aligned}
G_{n z} \frac{0.3 h^{3} d^{3}}{h^{2} d^{2}} \bar{\kappa}_{s n} & =\psi \sin \alpha+\varphi \cos \alpha \\
E_{s} \frac{d h^{3}}{12} \bar{\kappa}_{s} & =\varphi \sin \alpha-\psi \cos \alpha
\end{aligned}
$$

Eliminating $d$ from (37) leads to the condition for the shape of the neutral hole in terms of $\alpha(s)$ and $h(s)$. $\frac{0.3 G_{n z} 12^{3}(\varphi \sin \alpha-\psi \cos \alpha)^{3}}{E_{s}{ }^{2} h^{8} \bar{\kappa}_{s}^{2}+12^{2}\left((\varphi \sin \alpha-\psi \cos \alpha)^{2}\right.}=E_{s}(\psi \sin \alpha+\varphi \cos \alpha) \frac{\bar{\kappa}_{s}}{\bar{\kappa}_{s n}}$

The solution to equation (38), i.e. obtaining $\alpha(s)$, for a chosen $h$ (choosing the reinforcement thickness to be uniform and a certain multiple of the laminate thickness, say) will give the exact neutral hole shape. Finally, the reinforcement width distribution is determined from (37) as,

$$
d(s)=\frac{12(\varphi \sin \alpha-\psi \cos \alpha)}{E_{s} h^{3} \bar{\kappa}_{s}} .
$$

\section{Examples}

\subsection{Plate under biaxial planar loading}

In general, the stress function (as a solution to (2)) is in the form of a conic.

$$
\phi=\frac{1}{2}\left(P_{1} y^{2}+P_{2} x^{2}\right)+S x y+a x+b y+c
$$

where $P_{1}$ and $P_{2}$ are the tension forces per unit length in $x$ and $y$ directions respectivly, and $S$ is the shear force per unit length in the $x y$ plane. The constants $a, b$ and $c$ merely determine the position and the size of the hole. In order to have a closed hole, the condition $S^{2}-P_{1} P_{2}<0$ must be satisfied (a rotated conic is obtained in the presence of shear). If there is no shear then clearly, $P_{1}$ and $P_{2}$ must have the same sign. Further, in the absense of tensile (compressive) forces with shear alone, no closed holes are possible. For combined loads, the above inequality determines the existence of closed holes. If $x$ and $y$ axes are chosen as the principal axes, then the $S x y$ term in (40) is eliminated, and the remaining part gives an ellipse with lengths of major axes in the ratio $\sqrt{P_{1} / P_{2}}$. Since there are no restrictions on the constants $a, b, c$ a large variety of holes may be chosen.

Under the loading, as defined above, the plate resultants are $N_{x}=P_{1}, N_{y}=P_{2}, N_{x y}=S$. The strain state, then by (11) in terms of the material properties and the known loading, becomes

$$
\begin{aligned}
\epsilon_{x} & =A_{11}{ }^{*} P_{1}+A_{12}{ }^{*} P_{2}+A_{16}{ }^{*} S \\
\epsilon_{y} & =A_{12}{ }^{*} P_{1}+A_{22}{ }^{*} P_{2}+A_{26}{ }^{*} S \\
\epsilon_{x y} & =A_{16}{ }^{*} P_{1}+A_{26}{ }^{*} P_{2}+A_{66}{ }^{*} S
\end{aligned}
$$

From (5), (12) and (15) $d(s)$ is determined in terms of the known stress function and strain components as follows. 


$$
d(s)=\frac{\left[\left(\frac{\partial \phi}{\partial x}\right)^{2}+\left(\frac{\partial \phi}{\partial y}\right)^{2}\right]^{\frac{3}{2}}}{\bar{A}_{s s}\left[\epsilon_{x}\left(\frac{\partial \phi}{\partial y}\right)^{2}+\epsilon_{y}\left(\frac{\partial \phi}{\partial x}\right)^{2}-\epsilon_{x y}\left(\frac{\partial \phi}{\partial x}\right)\left(\frac{\partial \phi}{\partial y}\right)\right]}
$$

Equation (42) (along with (41)) gives the reinforcement width distribution in terms of the material properties and the stress function which is found in the uncut state of the plate.

As an example, consider a plate under forces $P_{1}$ and $P_{2}$ applied in the principal axes system as explained above. The stress function is $\phi=\frac{1}{2}\left(P_{1} y^{2}+P_{2} x^{2}\right)+c$. Then the hole shape (from (7)) is given by;

$$
P_{1} y^{2}+P_{2} x^{2}+2 c=0 .
$$

By using the relations $\frac{\partial \phi}{\partial x}=P_{2} x, \frac{\partial \phi}{\partial y}=P_{1} y$, and from (42), the width distribution $d(s)$ is found as,

$$
d(s)=\frac{\left[\left(P_{2} x\right)^{2}+\left(P_{1} y\right)^{2}\right]^{\frac{3}{2}}}{\bar{A}_{s s}\left[\epsilon_{x}\left(P_{1} y\right)^{2}+\epsilon_{y}\left(P_{2} x\right)^{2}-\epsilon_{x y} P_{1} P_{2} x y\right]} .
$$

If $y$ is solved from (43) and substituted into (44), along with (41), the width distribution is obtained as a function of $x$ only. A typical numerical example is given below.

Example: Lay-up: $[+40 /-40]_{2 s}$

Ply Properties: $E_{1}=133 \mathrm{GPa}, E_{2}=9 \mathrm{GPa}, G_{12}=3 \mathrm{GPa}$, $\nu_{12}=0.26$.

Ply Thickness: $1 \mathrm{~mm}$.

Shape of Hole (from above solution): Ellipse, $200 y^{2}+$ $150 x^{2}-15.10^{5}=0$

Equation describing the reinforcement Distribution:

$$
d(x)=\frac{\left(3.10 \times 10^{6}-75 \times x^{2}\right)^{\frac{3}{2}}}{1.82 \times 10^{8}-3.25 \times 10^{3} x^{2}}
$$

In the above solution the reinforcement thickness $(h)$ is chosen to be twice the thickness of the plate (see Figs.7a and $7 \mathrm{~b}$ ).

For some stacking sequences and fiber angles, the strain in the reinforcing member changes sign, which leads to a solution with very large reinforcement at that material point. This situation can be eliminated by changing either the stacking sequence or fiber angles in some layers, i.e., outer layers for example.
In the case of equal loading in the $x$ and $y$ directions a circular hole is obtained. The reinforcement distribution is easily found by setting $P_{1}=P_{2}$ in (44).

It should be mentioned that, in the case of having linearly varying loads at the edges of the plate, the most general form for $\phi$ is $x^{3}+y^{3}+a x+b y+c$, so that the shape of a neutral hole is, in general, a cubic. Here, the coefficients $a, b, c$ no longer refer directly to the position and size of the hole. However, the coefficients may be chosen specially so that $\phi$ may be factorized. This makes possible to obtain a closed form for the shape of the neutral hole. For example, considering $x^{3}+y^{3}-r^{2}(x+y)=(x+y)\left(x^{2}-x y+y^{2}-r^{2}\right)$ factorization, and choosing the second factor as the shape of the hole give a solution to the problem. The sectional area could then be determined by going through the same procedures as explained before in Section (2.1.2).

\subsection{Plate under bending moments \\ 3.2.1 Restricted solution}

In this case, let the plate be under pure bending moments $M_{1}$ and $M_{2}$ applied at the edges of the plate whose normals are $x$ and $y$ axes respectivly. $M_{t}$ is the twisting moment defined in the same system. The well known plate equlibrium equations give $M_{x}=M_{1}$, $M_{y}=M_{2}$ and $M_{x y}=M_{t}$. Then, from equations (19), (20) and (22) the functions $\varphi$ and $\psi$ are determined.

$$
\begin{aligned}
& \varphi=M_{2} x-M_{t} y+c_{1} \\
& \psi=M_{1} y-M_{t} x+c_{2}
\end{aligned}
$$

where $c_{1}$ and $c_{2}$ are constants of integration. Combining (46) and (26), and integrating gives the hole shape, as;

$$
\begin{aligned}
& \left(M_{2}-\lambda M_{t}\right) x^{2}+\left(M_{1}+\lambda M_{t}\right) y^{2} \\
& +\left[2 \lambda\left(M_{1}-M_{2}\right)-4 M_{t}\right] x y+2\left[c_{1}+\lambda\left(c_{2}+e\right)+f\right] x \\
& +2\left[c_{2}-\lambda\left(c_{1}+f\right)+e\right] y=C
\end{aligned}
$$

where $C$ is a constant. This equation is in the form of a conic. The constants $c_{1}, c_{2}, e, f$ do not affect the moment distribution, and they are chosen zero in (47) without loss of generality. Then, the governing equation for the hole becomes,

$$
\left(M_{2}-\lambda M_{t}\right) x^{2}+\left(M_{1}+\lambda M_{t}\right) y^{2}+2 \lambda\left(M_{1}-M_{2}\right) x y=C .
$$

The condition $\lambda^{2}\left(M_{1}-M_{2}\right)^{2}-\left(M_{2}-\lambda M_{t}\right)\left(M_{1}+\right.$ $\lambda M t)<0$ is necessary for a closed hole. The type of 
curves given by (47) with this restriction are the rotated ellipses. If $\beta$ is the angle of rotation measured from the positive $x$ axis, its value is given by,

$$
\tan 2 \beta=\frac{2 \lambda\left(M_{1}-M_{2}\right)}{M_{2}-M_{1}-2 \lambda M_{t}} .
$$

In order to determine the sectional area of the reinforcement, consider the state of curvature in the plate given by (29) as;

$$
\begin{aligned}
\kappa_{x} & =D_{11}{ }^{*} M_{1}+D_{12}{ }^{*} M_{2}+D_{16}{ }^{*} M_{t} \\
\kappa_{y} & =D_{12}{ }^{*} M_{1}+D_{22}{ }^{*} M_{2}+D_{26}{ }^{*} M_{t} \\
\kappa_{x y} & =D_{16}{ }^{*} M_{1}+D_{26}{ }^{*} M_{2}+D_{66}{ }^{*} M_{t} .
\end{aligned}
$$

Then, combining (24), (30), and (33) yields the reinforcement distribution, as the following.

$$
\begin{aligned}
\bar{d}(s) & =\frac{\left(1+\lambda^{2}\right)^{\frac{1}{2}}\left(\psi^{2}+\varphi^{2}\right)^{\frac{3}{2}}}{\bar{D}_{s s}\left[\kappa_{x} C_{1}{ }^{2}+\kappa_{y} C_{2}{ }^{2}+2 \kappa_{x y} C_{3}\right]} \\
C_{1} & =(\lambda \varphi-\psi) \\
C_{2} & =(\lambda \psi+\varphi) \\
C_{3} & =(\lambda \psi+\varphi)(\lambda \varphi-\psi)
\end{aligned}
$$

A typical numerical example case is stated below.

Example: $[+40 /-40]_{2 s}$

Ply Properties: $E_{1}=133 \mathrm{GPa}, E_{2}=9 \mathrm{GPa}, G_{12}=3 \mathrm{GPa}$, $\nu_{12}=0.26$.

Ply Thickness: $1 \mathrm{~mm}$.

Restriction: $\lambda=\mathbf{a}$ chosen constant.

Shape of Neutral Hole: Rotated ellipse, $150 x^{2}+200 y^{2}+$ $60 x y-75.10^{4}=0$

Reinforcement Distribution:

$$
\begin{aligned}
\bar{d}(s) & =\frac{(1.36)^{\frac{1}{2}}\left(4.10^{4} x^{2}+2.2510^{4}\right)^{\frac{3}{2}}}{\bar{D}_{s s}\left[\kappa_{x} C_{4}{ }^{2}+\kappa_{y} C_{5}{ }^{2}+2 \kappa_{x y} C_{6}\right]} \\
C_{4} & =(90 x-200 y) \\
C_{5} & =(120 y+150 x) \\
C_{6} & =(120 y+150 x)(90 x-200 y)
\end{aligned}
$$

where $\bar{D}_{11}=5700.74, \kappa_{x}=0.0620703, \kappa_{y}=0.0627094$ and $\kappa_{x y}=0.0427512$. Here, the reinforcement thickness is chosen to be twice the thickness of the plate (see Figs. $8 \mathrm{a}$ and $8 \mathrm{~b}$ ).

\subsubsection{Plate bending incorporating reinforcement kinematics}

In this example, the same bending problem considered above is solved numerically, by considering reinforcement kinematics (as described in section 2.3) to arrive at an exact solution for the hole shape and reinforcement distribution. Corresponding to the previously solved problem, we have,

$$
\begin{aligned}
& \varphi=M_{2} x \\
& \psi=M_{1} y
\end{aligned}
$$

When these values are substituted in equation (38) and after some algebraic manipulation, a $7^{\text {th }}$ order polynamial in $\tan \alpha$ is obtained. The roots of this polynamial are obtained to describe the shape of the neutral hole. The procedure is as follows. A starting point in the plate is identified. Then the equations are cast into cylidrical polar coordinates, where, a radius vector $R(\theta)$ is chosen to describe the equation for the neutral hole as $R=R(\theta)$. At some initial point $R_{0}, \theta_{0}$, in the $R-\theta$ plane the roots of equation (38) are used to obtain $\frac{d R}{d \theta}$. Then, $\theta$ is incremented in a forward difference scheme to obtain a new $R$. At this $R, \theta$, the above step is repeated. Inspection of the terms in (38) cast in the $R-\theta$ plane reveals that $R(\theta)=R(\theta+2 \pi)$, thus describing a closed hole. In fact, $(38)$ can be used to determine the conditions under which a closed hole is possible. Once the neutral hole shape is determined, the reinforcement distribution is easily computed from (39). To demonstrate this procedure the following numerical example is presented.

Example: $[+40 /-40]_{2 s}$

Ply Properties: $E_{1}=133 \mathrm{GPa}, E_{2}=9 \mathrm{GPa}, G_{12}=3 \mathrm{GPa}$, $G_{23}=5 \mathrm{GPa}, \nu_{12}=0.26$.

Ply Thickness: $1 \mathrm{~mm}$.

Shape of Neutral Hole: The above explained solution scheme is implemented to obtain $\alpha(s)$.

Reinforcement Distribution: from equation (39) with prescribed thickness (chosen to be the same as the laminate thickness, $8 \mathrm{~mm}$.)

Results : Presented in Figs. 9a and $9 \mathrm{~b}$.

\section{Conclusions}

In this paper we have presented a method to design the shape of a cutout and the cutout edge reinforcement 
distribution for a symmetrically laminated plate, when it is remotely loaded by uniform planar loads or uniform bending moments. It should be noted that in the realm of classical linear plate theory, the in-plane and out-ofplane plate responses are uncoupled. This has led us to two different solutions as expected.

In the laminated plates we studied, the fibers are straight in each layer of the plate, and they are tangential to the edge of the cutout in the reinforcement. This is not a restriction, as the solution presented could very well accommodate other types of constitutive models for the reinforcement. Classical laminate theory to model the laminate and technical beam theory to model the reinforcement are used. In many practical constructions, it has been shown before that the bending-stretching coupling diminishes as the laminate gets thicker. We have developed the solutions for these unsymmetrical layups (not reported here) and found that the material coupling has a small effect on the reinforcement distribution in many cases. Neglecting this material coupling and using the procedures developed here leads to a solution for unsymmetrically laminated plates. The case of strong coupling (few layers) is reported elsewhere [9].

Example results have been presented for two problems. For purposes of comparison, the same lay-up and material properties have been used in both cases. For the case of remotely applied moments, two different solution methods are presented. One is simple and approximate, relying on practicality, while the other is more refined and exact within the context of classical laminate theory. As mentioned in the introduction, all previous attempts to obtain a neutral hole have been done without accounting for flexural plate response, and assuming material isotropy. Here, both of these restrictions are relaxed. The solutions reported here are novel and their extension to the more complex case of laminated cylidrical panels and laminated shells is underway.

\section{Acknowledgments}

The first author is grateful to the Turkish Government for the financial assitance through a Graduate Research Assistantship. The authors are appreciative of the support received from the Rackham Graduate School, Univ. of Michigan, throughout this study.

\section{References}

[1] Mansfield, E.H., "Neutral Holes in Plane SheetReinforced Holes Which are Elastically Equivalent to the Uncut Sheet," Quarterly Journal of Mechan- ics and Applied Mathematics, London, England, Vol.VI, Part 3, 1953, pp. 370-378.

[2] Lekhnitskii, S.G., "Anisotropic Plates", translated by Tsai, S.W., and Cheron, T., Gordon and Breach, New York, 1968.

[3] Lekhnitskii, S.G., "Theory of Elasticity of an Anisotropic Body", translated by Fern, P., and Brandstatter, J.J., Holden-Day, San Fransisco, Calif., 1963.

[4] Savin, G.N., "Stress Distribution Around Holes", NASA TT F-607, Nauka Dumka Press, 1968.

[5] Richards, R.,Jr., and Bjorkman, G.S.,Jr., "Neutral Holes; Theory and Design", Journal of the Engineering Mechanics Divison ASCE, Vol. 108, 1982, pp.945-960.

[6] Whitney, J.M., "Structural Analysis of Laminated Anisotropic Plates", Technomic Publishing Co., Inc., Penn.,1987.

[7] Timoshenko, S., Woinowsky-Krieger, S., "Theory of Plates and Shells", McGraw-Hill, New York, 1959.

[8] Mansfield, E.H., "The Bending and Stretching of Plates", Macmillan Company, New York, 1964, pp. $3-20$.

[9] Senocak, E and Waas, A., "Neutral holes in laminated plates", The Univ. of Michigan, Dept. of Aeropsace Engineering Report SM92-1, 1992. 


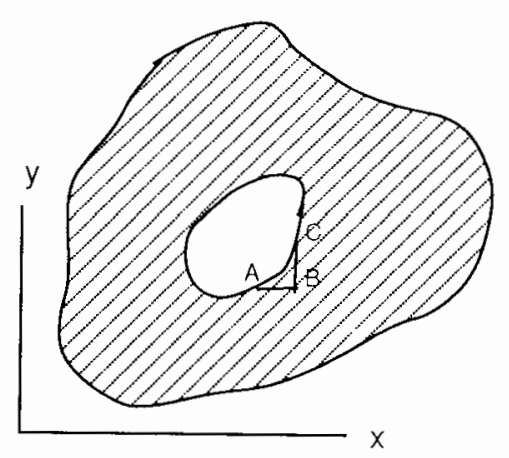

Figure 1: Plate with a cutout.

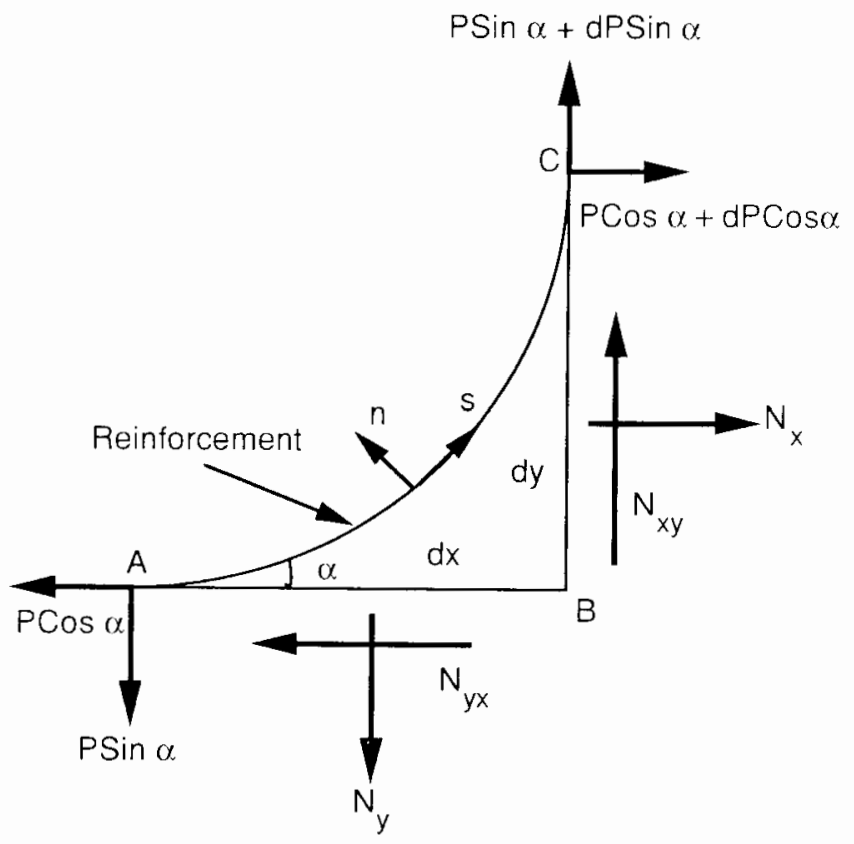

Figure 2:Forces on the small element 'ABC' indicated in figure 1.

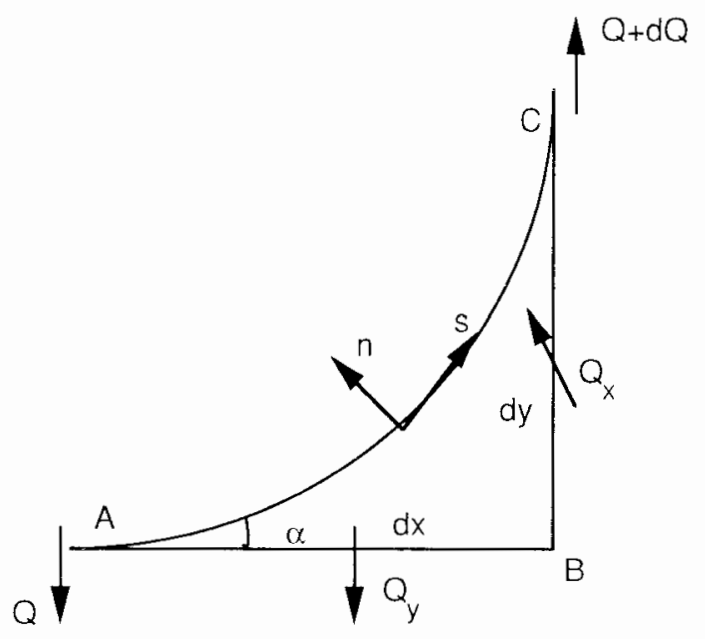

Figure 3: Out-of-plane forces.

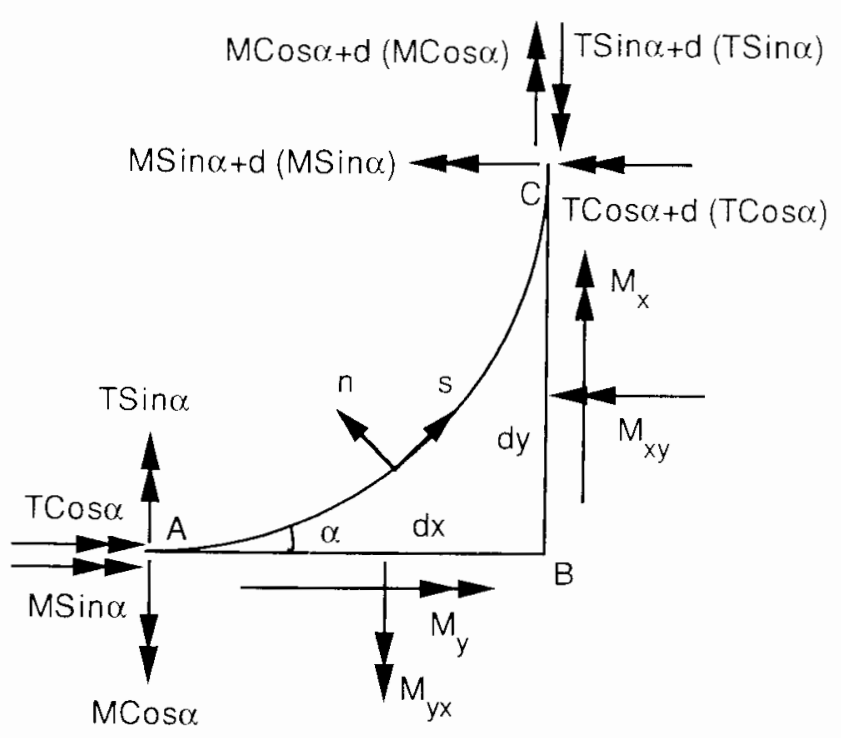

Figure 4: Moments acting on the element $A B C$. 


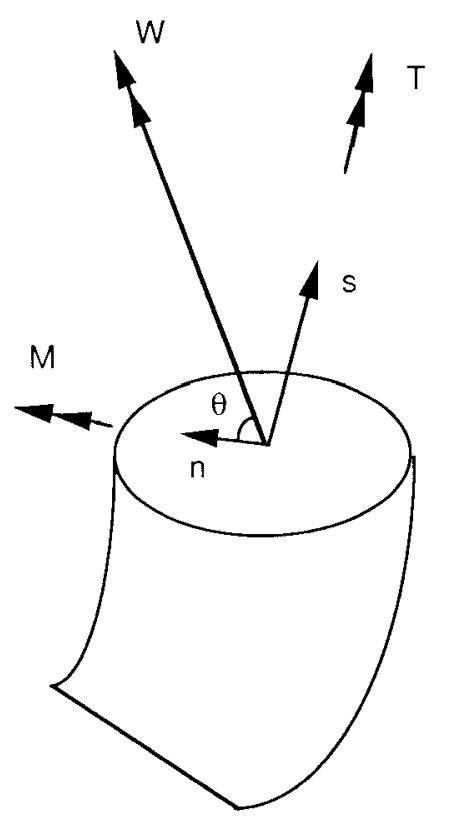

$F$ igure 5: Moments in the reinforcement.

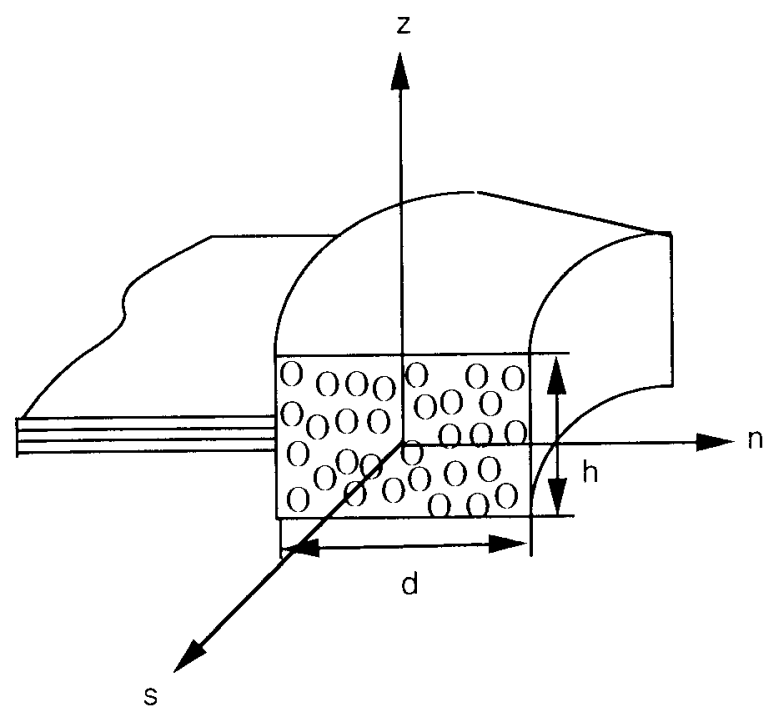

Figure 6: Coordinates used to define reinforcement properties.

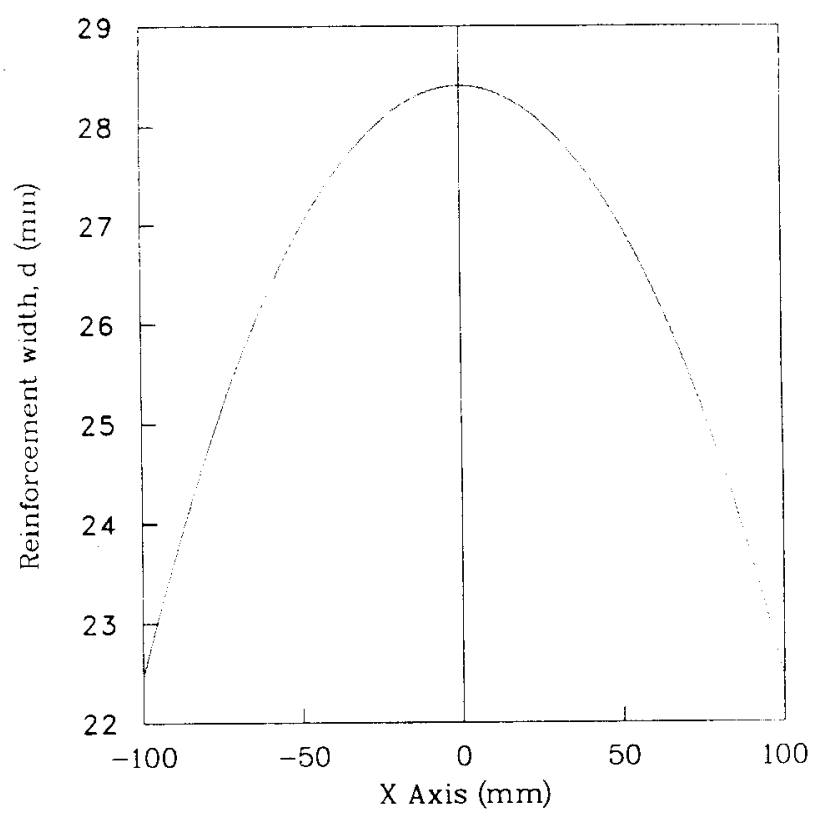

Figure 7a: Reinforcement distribution for planar loading.

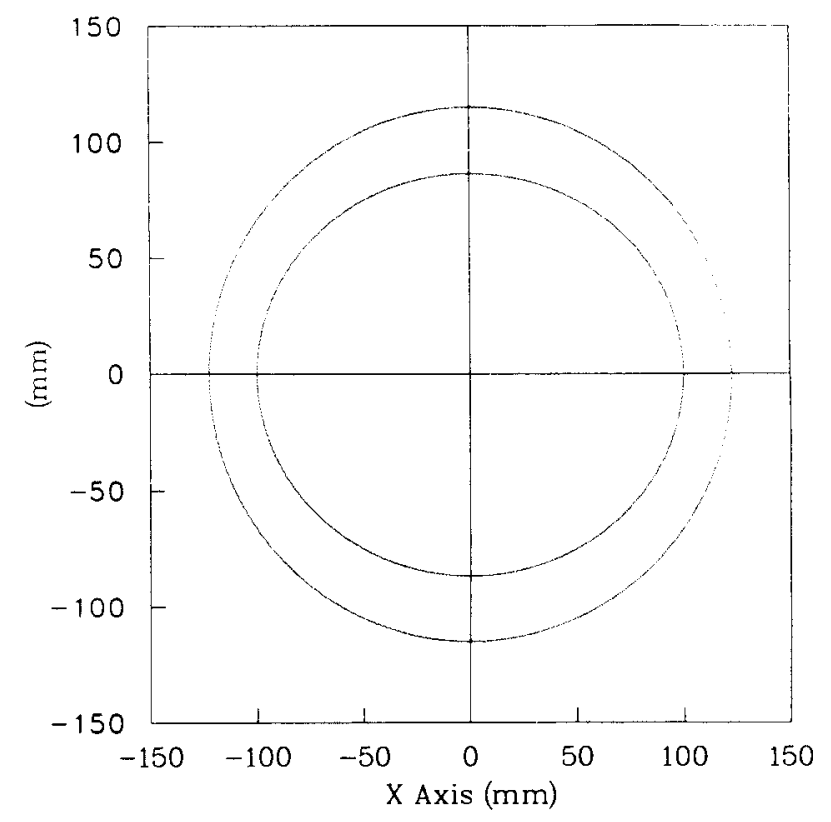

Figure 7b: Reinforcement distribution and hole shape for planar loading. 


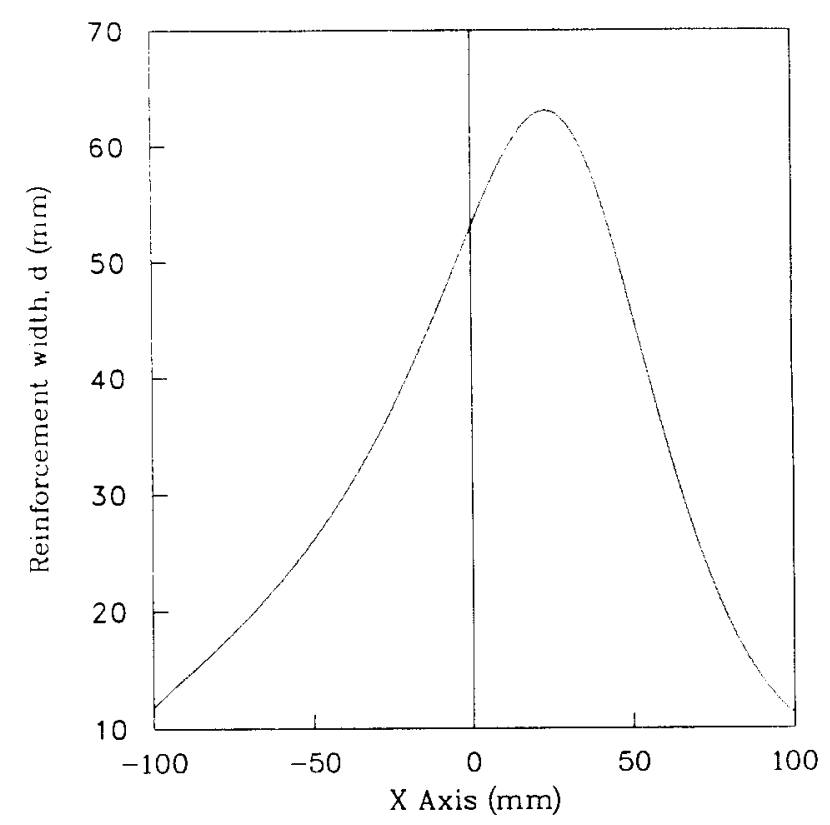

Figure 8a: Reinforcement distribution for moment loading; restricted solution.

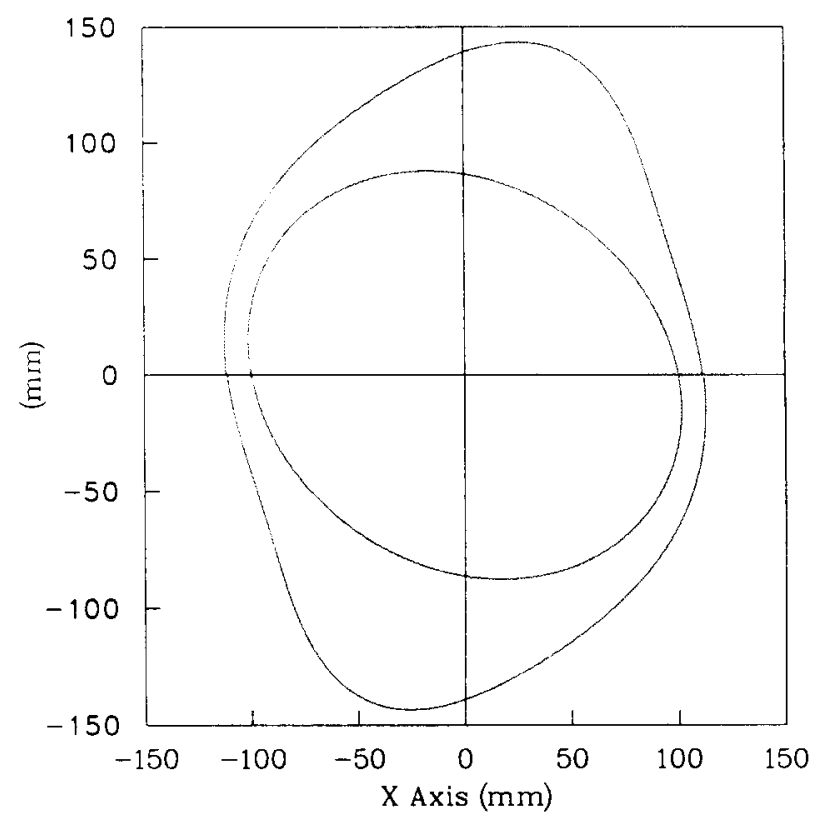

Figure 8b: Reinforcement distribution and hole shape for moment loading; restricted solution.

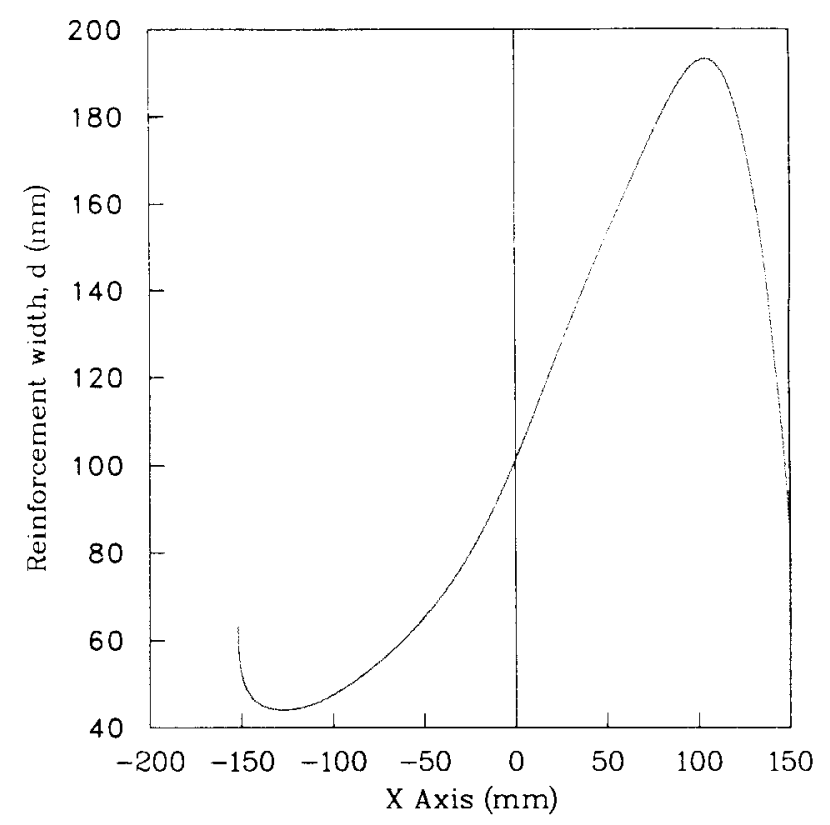

Figure 9a: Reinforcement distribution for moment loading; exact solution.

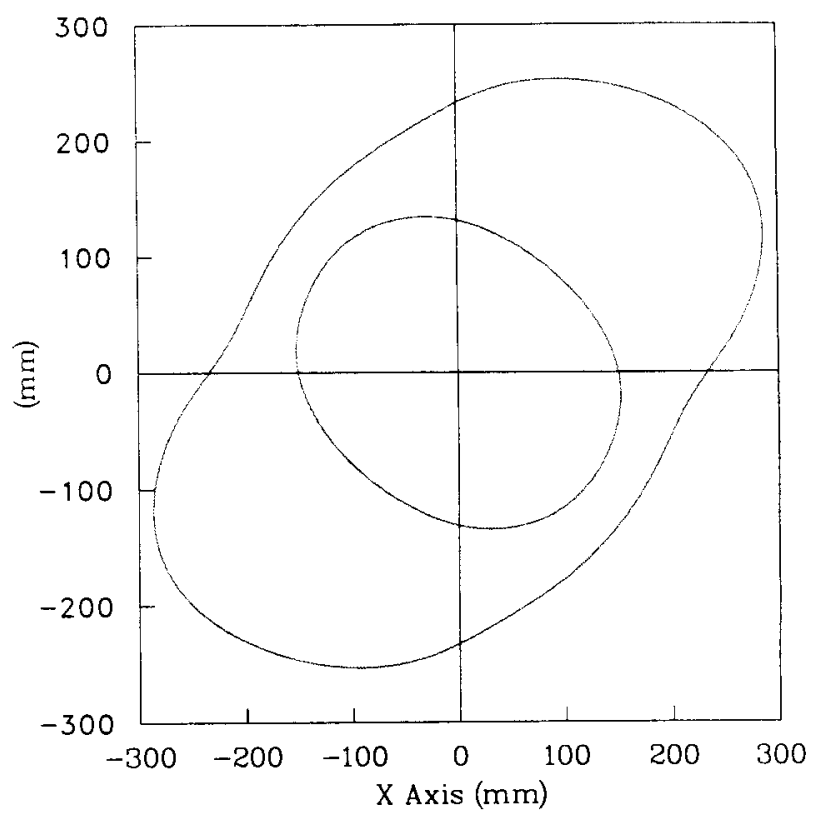

Figure 9b: Reinforcement distribution and hole shape for moment loading; exact solution. 